\title{
Evolution equation of moving defects: dislocations and inclusions
}

\author{
Xanthippi Markenscoff
}

Received: 24 November 2009 / Accepted: 18 March 2010 / Published online: 24 August 2010

(C) The Author(s) 2010. This article is published with open access at Springerlink.com

\begin{abstract}
Evolution equations, or equations of motion, of moving defects are the balance of the "driving forces", in the presence of external loading. The "driving forces" are defined as the configurational forces on the basis of Noether's theorem, which governs the invariance of the variation of the Lagrangean of the mechanical system under infinitesimal transformations. For infinitesimal translations, the ensuing dynamic $\mathbf{J}$ integral equals the change in the Lagrangean if and only if the linear momentum is preserved. Dislocations and inclusions are "defects" that possess self-stresses, and the total driving force for these defects consists only of two terms, one expressing the "self-force" due to the self-stresses, and the other the effect of the external loading on the change of configuration (Peach-Koehler force). For a spherically expanding (including inertia effects) Eshelby (constrained) inclusion with dilatational eigenstrain (or transformation strain) in general subsonic motion, the dynamic $\mathrm{J}$ integral, which equals the energy-release rate, was calculated. By a limiting process as the radius tends to infinity, the driving force (energy-release rate) of a moving half-space plane inclusion boundary was obtained which is the rate of the mechanical work required to create an incremental region of eigenstrain (or transformation strain) of a dynamic phase boundary. The total driving force (due to external loading and due to self-forces) must
\end{abstract}

X. Markenscoff ( $\square)$

Department of Mechanical and Aerospace Engineering, UCSD, La Jolla, CA 92093, USA

e-mail: xmarkens@ucsd.edu be equal to zero, in the absence of dissipation, and the evolution equation for a plane boundary with eigenstrain is presented. The equation applied to many strips of eigenstrain provides a system to solve for the position/ evolution of strips of eigenstrain.

Keywords Eshelby inclusions - Eigenstrain · Transformation strain - Dynamic phase boundary . Evolution equation · Dynamic J integral

\section{Introduction}

In a 1953 paper, Eshelby (1953) obtained the equation of motion for a dislocation, the expression containing two terms (in the absence of dissipation), one accounting for the self-force (due to its own radiated field) which involves the acceleration, and one due to external loading (Peach-Koehler force). This equation of motion is the dynamic $J$ integral (Atkinson and Eshelby 1968; Freund 1972; Eshelby 1970), which for a dislocation in generally accelerating motion has been further evaluated by Ni and Markenscoff (2008), and Markenscoff and Huang (2009) at the transition from subsonic to supersonic motion. The dynamic $J$ integral is obtained by the application of Noether's theorem to the Lagrangean functional for invariance under infinitesimal translation of the defect, and a new interpretation is given (Gupta and Markenscoff in preparation), analogously to the one for static deformations in Gupta and Markenscoff (2008). The dynamic $J$ 
integral has been applied to dynamic crack propagation (Eshelby 1970; Freund 1990; Maugin 1990) and is equivalent to the energy-release rate (Eshelby 1970; Freund 1990; Maugin 1990), expressed by the variation of the Hamiltonian functional in Noether's theorem, with conditions of equivalence between variation of the Lagrangean and the Hamiltonian obtained in Gupta and Markenscoff (in preparation). The physical meaning of the energy-release rate is, for the dislocation, the energy-rate required to produce an incremental slip region, and, for the crack, an incremental opening displacement. Recently (Markenscoff and Ni 2010a), the dynamic $J$ integral (and energy-release rate) was evaluated when the defect is an expanding Eshelby inclusion, and an incremental volume of eigenstrain is produced. It reveals the structure of the self- force and its dependence on velocity and motion, in the case of a spherical and plane boundary (with dilatational eigenstrain) in general motion. For a purely mechanical system, since the defect moves in the presence of external loading, when the total Lagrangean functional (including the loading) is applied in Noether's theorem, in the vanishing of total dynamic $J$ integral the relation between loading and defect motion ensues necessarily. This is implemented here for a spherical and plane expanding inclusion boundary under external loading of uniform hydrostatic tension and uniaxial tension, respectively.

\section{Noether's theorem and the dynamic $J$ integral}

Consider the Lagrangean $L=W-T$, with $W$ denoting the strain energy and $T$ the kinetic energy per unit volume $T=\frac{1}{2} \rho \dot{u}_{i}^{2}$, with the density $\rho$ constant, and the functional

$\Pi_{L}\left(u_{i, j}, \dot{u}_{i}\right)=\int_{t_{1}}^{t_{2}} \int_{\Omega}\left(W\left(x_{i}, u_{i, j}\right)-T\left(\dot{u}_{i}\right)\right) d V d t$

The application of Noether's theorem (see e.g. Gelfand and Fomin 2000) to the functional

$$
\prod\left(u_{i}, u_{i, j}, \dot{u}_{i}\right)=\int_{t_{1}}^{t_{2}} \int_{\Omega} L\left(x_{i}, u_{i}, u_{i, j}, \dot{u}_{i}\right) d V d t
$$

under transformations $y_{\alpha}=x_{\alpha}+\phi_{\alpha}+O\left(\varepsilon^{2}\right)$ and $v_{i}=$ $u_{i}+\psi_{i}+O\left(\varepsilon^{2}\right)$, where $\alpha=1-4$ and $x_{4}=t$ (therefore $\dot{u}_{i} \equiv u_{i, 4}$ ), was performed in Gupta and Markenscoff (in preparation), and for infinitesimal translations $\phi_{i}=$ $\varepsilon_{i}, \phi_{4}=0$ and $\psi_{i}=0$, the variation of Eq. 1 was obtained (Gupta and Markenscoff in preparation):

$$
\begin{aligned}
\delta_{x} \Pi_{L}= & \varepsilon_{j} \int_{t_{1}}^{t_{2}} \int_{\partial \Omega} E_{i j} n_{i} d A d t+\varepsilon_{j} \int_{t_{1}}^{t_{2}} \int_{\Omega} \frac{d}{d t}\left(\rho u_{k, j} \dot{u}_{k}\right) d V d t \\
& -\varepsilon_{k} \int_{t_{1}}^{t_{2}} \int_{\Omega}\left(-\sigma_{i j, j}+\rho \ddot{u}_{i}\right) u_{i, k} d V d t
\end{aligned}
$$

with the first two terms on the right being defined as the dynamic $J$ integral and where $E_{i j}$ is the dynamic energy-momentum tensor

$E_{i j}=\left((W-T) \delta_{i j}-u_{k, j} \frac{\partial W}{\partial u_{k, i}}\right)$

and the second term (volume integral) on the righthand-side of Eq. 2 is called "pseudomomentum". It was proved in Gupta and Markenscoff (in preparation) that the variation of the Lagrangean functional is equal to the dynamic $J$ integral if and only if linear momentum is conserved, or, in other words, for linear momentum to be conserved, the variation of the Lagrangean must equal to the dynamic $J$ integral in any rearrangement of materials points in the translation of the defect (for statics, see Gupta and Markenscoff (2008)). It may be noted that for a "singularity" that is a jump discontinuity (and, hence, integrable), the variation of the Lagrangean is equal to the variation of the Hamiltonian (energy-release rate), which is also true for cracks (Eshelby 1970; Freund 1990; Maugin 1990), for which the integrals involved exist as Cauchy Principal Values.

\section{The equation of motion of a dislocation and an eshelby inclusion boundary}

In an early paper, Eshelby (1953) gave the equation of motion of a screw dislocation, which consists of two terms: the Peach-Koehler force (on the left hand side) and the self-force due to inertia effects on the right:

$$
\begin{aligned}
F_{x}= & b p_{z y}{ }^{A}=\left(1-\dot{\xi}^{2} / c^{2}\right)^{-\frac{3}{2}}\left(\rho b^{2} / 4 \pi\right) \\
& \times\{\ln f(t)\} \partial^{2} \xi / \partial t^{2}+g(t)
\end{aligned}
$$

where $b$ denotes the Burgers vector, $\xi(t)$ the motion, and $c$ the shear-wave speed. It may be noticed that there is no term involving both the applied stress and the motion. The self-force terms depends on the acceleration $\partial^{2} \xi / \partial t^{2}$, with the functions $f(t)$ and $g(t)$ in Eq. 3 remaining undetermined for general motion, and being recently calculated by $\mathrm{Ni}$ and Markenscoff (2008) for general screw dislocation motion with arbitrary acceleration. 
The coefficient of the logarithmic singularity in the near-field can be calculated either from the full solution of the generally moving dislocation by singular asymptotics of integrals (Callias and Markenscoff 1988), or by the dynamic energy-momentum tensor as a field equation (Ni and Markenscoff 2009).

The equation of motion is sought here for an expanding Eshelby inclusion boundary, with the first problem being the spherical inclusion with dilatational eigenstrain, by a limiting procedure from which the plane boundary is obtained as the radius tends to infinity. For a dynamically expanding (in general subsonic motion) spherical Eshelby inclusion with dilatational eigenstrain the radiated fields have been calculated (Markenscoff and Ni 2010a) on the basis of the dynamic Green's function

$$
\begin{aligned}
u_{i}(x, t)= & \int_{-\infty}^{\infty} d t^{\prime} \int_{\partial B_{t}} C_{j k l m} \delta_{l m} \varepsilon^{*} n_{k}\left(x^{\prime}\right) \\
& \times G_{i j}\left(x-x^{\prime}, t-t^{\prime}\right) d S_{X}
\end{aligned}
$$

where the boundary of the spherical inclusion is expanding according to time dependent radius $R(t)=$ $R_{0}+l(t)$ for $t \geq 0$ in a monotonically increasing subsonic motion. The calculated fields satisfy the Hadamard jump conditions on the moving boundary (Markenscoff and Ni 2010a) and yield the static Eshelby solution when calculated from time $t=-\infty$ to $t=0$.

The energy-release rate $\dot{E}$ is calculated on a contour surrounding the interface moving with $v_{n}$ and shrinking onto it from the classical expression (Freund 1972)

$\dot{\mathrm{E}}=\lim _{\varepsilon \rightarrow 0} \int_{S_{\in}}\left[n_{j} \sigma_{i j} \dot{u}_{i}+v_{n}(W+T)\right] d S$

which, with the use of the Hadamard jump conditions, and dividing by the velocity of the moving boundary, yields the "driving force" on it per unit area (Markenscoff and Ni 2010a; Stolz 2003):

$f=[[W]]-\left\langle\sigma_{i j}\right\rangle\left[\left[\frac{\partial u_{i}}{\partial x_{j}}\right]\right]$

with the double brackets denoting jumps and the $\langle\cdot\rangle$ averages. This "driving force" expression accounts for the mechanical work needed to create an additional volume of eigenstrain, no matter which is the source of creation of this eigenstrain, which will be accounted for in a total energy-rate balance equation.

For the spherically expanding inclusion the energyrelease rate (self-force to create an incremental region of eigenstrain) has been evaluated (Markenscoff and $\mathrm{Ni}$ 2010a) in the form

$$
\begin{aligned}
f= & \varepsilon^{*}(3 \lambda+2 \mu)\left(\left\langle\frac{\partial u_{r}}{\partial r}\right\rangle+2\left\langle\frac{u_{r}}{r}\right\rangle\right)-\frac{3}{2}(3 \lambda+2 \mu) \varepsilon^{* 2} \\
= & -\frac{2 \mu(3 \lambda+2 \mu) \varepsilon^{* 2}}{(\lambda+2 \mu)}-\frac{(3 \lambda+2 \mu)^{2} \varepsilon^{* 2}}{2(\lambda+2 \mu)}\left[\frac{a \dot{l}(t)}{\left(a^{2}-\dot{l}^{2}(t)\right)}\right] \\
& +H\left(a t-\left(R(t)+R_{0}\right)\right) \frac{(3 \lambda+2 \mu)^{2} \varepsilon^{* 2}}{2(\lambda+2 \mu)} \\
& \times\left.\left[\frac{R\left(\tau_{1}\right)}{R(t)}\left(\frac{\dot{l}\left(\tau_{1}\right)}{a+\dot{l}\left(\tau_{1}\right)}\right)\right]\right|_{r=R(t)}
\end{aligned}
$$

where, the first term is the static contribution, coinciding with the static Eshelby inclusion self-force (independent of the radius of the sphere) obtained by Gavazza (1977) and Eshelby (1977), the second one, depending on the velocity of the moving boundary, is due to inertia, while the third one is the contribution from the jump in the eigenstrain at the furthest away point at the back of the inclusion that had the time to reach the front boundary.

In the presence of loading due to an externally applied field of uniform tensile radial expansion $\sigma^{a p p l}$, Eq. 6, with all the interaction energy terms included, will yield the additional term $3 \varepsilon^{*} \sigma^{a p p l}$, which is the counterpart of the Peach-Koehler force, with no terms coupling the applied loading to boundary velocity appearing. The equation of motion for the spherically expanding boundary (constrained) will be the total driving force (the sum of Eq. 7 and $3 \varepsilon^{*} \sigma^{a p p l}$ ) to vanish. It may be seen that the derivative of $\sigma^{a p p l}$ with respect to the boundary velocity is positive, implying an increasing external force for increasing velocity, i.e., stability.

The half-space inclusion (constrained by the matrix) can be obtained from the spherical one by a limiting process as the radius tends to infinity. In the twodimensional static case of circular inclusions and inhomogeneities, the limiting procedure was applied by Dundurs and Markenscoff (2009) and strip solutions were obtained, as well as the corresponding driving forces. The fields obtained by the limit correspond to the Eshelby solution (Eshelby 1957) for the interior domain and the Hill (1961) jump conditions, and are the minimum energy solution. Superposed selfequilibrated compatible fields at infinity will increase the energy of the system and were called by Dundurs and Markenscoff (2009) "rogue states". In the expanding inclusion case, by taking the limit of the radius of the inclusion tending to infinity, a moving 
Fig. 1 The limiting tractions of the spherically expanding Eshelby inclusion as the radius tends to infinity (plane moving boundary)

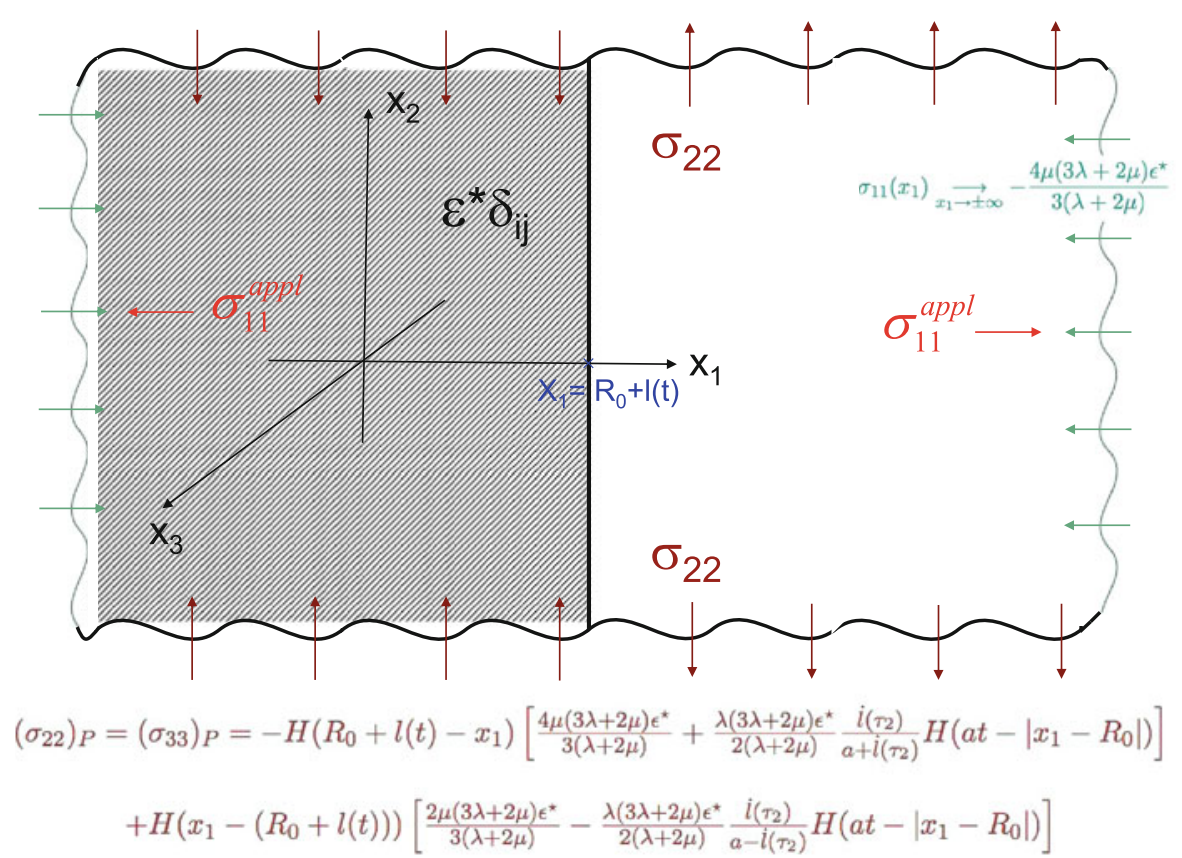

plane boundary of a constrained Eshelby inclusion was obtained (Markenscoff and Ni 2010a) with timedependent tractions on the lateral boundaries at infinity (Fig. 1), which ensure compatibility of the deformation at the interface. These fields are the unique limit of the linear elasticity problem, and the boundary value elastodynamic problem depicted in Fig. 1, with time dependent tractions on the boundaries at infinity those obtained by the limit of the sphere in Markenscoff and $\mathrm{Ni}$ (2010a), has a unique solution for the plane boundary to be at the position $l(t)$. The initial condition for the plane moving boundary is the limit of the spherical Eshelby inclusion, and is the unique minimum energy solution for a static plane inclusion boundary.

The self-force on the plane moving boundary with dilatational eigenstrain is calculated (Markenscoff and Ni 2010a), and with an additional uniaxial applied stress field present, the total driving force, including all the interaction energies in Eq. 6, yields the equation of motion for a moving plane inclusion boundary (with dilatational eigenstrain):

$$
\begin{aligned}
& -\frac{2 \mu(3 \lambda+2 \mu) \varepsilon^{* 2}}{(\lambda+2 \mu)}-\frac{(3 \lambda+2 \mu)^{2} \varepsilon^{* 2}}{2(\lambda+2 \mu)}\left[\frac{a \dot{l}(t)}{\left(a^{2}-\dot{l}^{2}(t)\right)}\right] \\
& +\sigma_{11}^{a p p l} \varepsilon^{*}=0
\end{aligned}
$$

The last term is the counterpart of the Peach-Koehler force, where now the eigenstrain is in place of the
Burgers vector. Equation 8 implies that the inclusion boundary does not move until the applied stress reaches the value so that the last term cancels the first static one (Eshelby 1977) at which point it becomes unstable. Subsequently, the inclusion boundary starts moving, and Eq. 8 relates the applied stress to the velocity. Equation 8 is the equation of motion, or evolution equation, or "kinetic relation" for the plane (constrained) inclusion boundary with dilatational eigenstrain in a uniaxial tensile field. Stability of the motion is governed by the derivative of the applied stress with respect to the boundary velocity, which for subsonic motion is positive.

The above equation of motion, or evolution, relates the applied stress to the velocity of the motion in the presence only of mechanical effects; dissipation will make the right hand side a function dependent on velocity, experimentally determined. While both terms (the inertia and dissipation one) depend on the velocity, the contribution of each of them may be separated in the analysis of experimental data.

The application of the energy-momentum tensor as driving force for interfaces as a particular kind of defect, the other being the crack and the dislocation, was presented by Eshelby (1970); it may be called the "Eshelby principle for an interface". Quoting from Eshelby (1970): 


$$
F=[W]-T \cdot\left[\frac{\partial u}{\partial n}\right] \quad \text { (47) in Eshelby (1970) }
$$

Equation (45) (above) can be used to find the equilibrium position of phase and twin boundaries, in the presence of stresses produced by the transformation itself, or applied externally, or both. Since equation (45) must be zero for any small $\delta \xi$, the boundary must take up shape for which equation (47) is zero all along it.

which, in the presence of inertia, means that the vanishing of the total (including external loading) driving force in Eq. 8 determines the boundary position.

\section{Motion/evolution of interacting Strips of Eigenstrain}

While the results obtained here are for dilatational eigenstrain only, the same principles of the dynamic $J$ integral determining the equation of motion can be also applied to inclusions with general eigenstrains, as argued by Eshelby (1970) for static phase boundaries. The self-force of the spherical inclusion has also a term (the last in Eq. 7) from the contribution of the farthest away point at the back of the inclusion (see figure 1 in Markenscoff and Ni (2010a)), which has the time to reach the front boundary. The solution of a shrinking spherical inclusion (and the limit of it for a plane boundary) was not obtained here. This will result in the solution of the spherical shell with eigenstrain (with the two boundaries moving in independent general motions), which will yield in the limit the solution for the general motion of expanding/shrinking strips. Recently Markenscoff and Ni (2010b) obtained the energy-release rates/"driving force" on the plane boundaries of a strip inclusion of general eigenstrain moving in general subsonic motion, including shear. Similarly to the spherically expanding inclusions with driving force given by Eq. 7, in the expanding/ shrinking strips of eigenstrains treated in Markenscoff and Ni (2010b), the discontinuities in the strain from the back boundary can contribute at later times on the front boundary, in addition to its own "selfforce". For multiple strips, for each plane boundary of a strip, an equation of motion like Eq. 8 will be formulated with contributions from all other boundaries and the applied loading, as well as a dissipation term, and this will involve the motion $l_{i}(t)$ of all boundaries.
The ensuing system of non-linear ode's will provide the solution for the motion of strip boundaries, much like Dislocation Dynamics codes that account for the interaction of dislocations and model the micromechanics of dynamic plasticity. As long as the number of boundaries is finite, this will hold (L.B. Freund, private communication). It may be noted that a recent reference by Yang et al. (2009) modeling phase transformations also considers constrained (spherical) Eshelby inclusions.

\section{Conclusions}

The dynamic $J$ integral, equivalent to the energyrelease rate (for integrable singularities), provides the framework for the relation between applied force and defect motion in the presence of mechanical effects (evolution equation). The equations of motion are analogous for inclusion boundaries and dislocations, and are presented here for a spherical and a plane boundary with dilatational eigenstrain in general subsonic motion. As it appears from Eqs. 1 and 8 respectively, the self-force for the dislocation (needed to create an additional slip region) depends on the acceleration, while, for the plane inclusion boundary, the self-force (needed to create an incremental region of eigenstrain) depends on the velocity only, and, hence, the dislocation has an "effective mass", while the phase boundary does not. A connection between the self-forces of dislocation and inclusion boundaries remains an open question for further investigation.

Acknowledgments The support of National Science Foundation grant \# CMS 0555280 is gratefully acknowledged.

Open Access This article is distributed under the terms of the Creative Commons Attribution Noncommercial License which permits any noncommercial use, distribution, and reproduction in any medium, provided the original author(s) and source are credited.

\section{References}

Atkinson C, Eshelby JD (1968) The flow of energy into the tip of a moving crack. Int J Fract Mech 4:3-8

Callias C, Markenscoff X (1988) Singular asymptotics of integrals and the near-field radiated from nonuniformly moving dislocations. Arch Ration Mech Anal 102:273-285

Dundurs J, Markenscoff X (2009) Stress fields and Eshelby forces on half-plane inhomogeneities and strip inclusions meeting a free surface. Int J Sol Str 46:2481-2485 
Eshelby JD (1953) The equation of motion of a dislocation. Phys Rev 90:248-255

Eshelby JD (1957) The determination of the elastic field of an ellipsoidal inclusion and related problems. Proc R Soc Lond A 241:376-396

Eshelby JD (1970) Energy relation of the energy-momentum tensor in continuum mechanics. In: Kanninen MF et al (eds) Inelastic behavior of solids, pp 77-115. Also: in Markenscoff X, Gupta A (eds) (2006) J.D. Eshelby's collected works: the mechanics of defects and inhomogeneities. Springer, pp 603-641

Eshelby JD (1977) Lecture on the elastic energy-momentum tensor. In: Markenscoff X, Gupta A (eds) (2006) Collected works of J.D. Eshelby: the mechanics of defects and inhomogeneities. Springer, Netherlands, pp 907-931

Freund LB (1972) Energy-flux into the tip of an extending crack in an elastic solid. J Elast 2:293-299

Freund LB (1990) Dynamic fracture mechanics. Cambridge University Press, Cambridge

Gavazza SD (1977) Forces on pure inclusion and Somigliana dislocations. Scr Metall 11:979-981

Gelfand IM, Fomin SV (2000) Calculus of variations. Dover, Mineola

Gupta A, Markenscoff X (2008) Configurational forces as dissipative mechanism: a revisit. C R Mec 336:126-131

Gupta A, Markenscoff X (in preparation) An interpretation of the dynamic $J$ integral

Gupta A, Markenscoff X (in preparation) Variations of the Hamiltonian of a moving defect
Hill R (1961) Discontinuity relations in mechanics of solids. In: Sneddon IN, Hill R (eds) Progress in solid mechanics. North-Holland, Amsterdam

Markenscoff X, Huang S (2009) The energetic of dislocations accelerating/decelerating through the shear wave-speed barrier. Appl Phys Lett 94

Markenscoff X, Ni L (2010a) The energy-release rate and selfforce of dynamically expanding spherical and plane inclusion boundaries with dilatational eigenstrain. J Mech Phys Sol 58:1-11

Markenscoff X, Ni L (2010b) Driving forces and radiated fields for expanding/shrinking half-space and strip inclusions with general eigenstrain. Q Appl Math (in press)

Maugin GA (1990) Material inhomogeneity in elasticity. Chapman and Hall, London

Ni L, Markenscoff X (2008) The self-force and effective mass of an accelerating dislocation. I. Screw dislocation. J Mech Phys Sol 56:1348-1379

Ni L, Markenscoff X (2009) The logarithmic singularity of a generally accelerating dislocation from the dynamic energymomentum tensor. Math Mech Sol 14:38-51

Stolz C (2003) Energetical approaches in nonlinear mechanics. Lecture Notes 11, Center of Excellence for Advanced Materials and Structures, Warsaw

Yang SY, Escobar J, Clifton RJ (2009) Computational modeling of stress waves induced martensitic transformations. Math Mech Sol 14:220-257 